\title{
Effects of Brown Rice and Germinated Brown Rice Extracts from Thai Rice Cultivars (PL2 and KDML105) on Adipogenic, Adipocytokine, and Antioxidant Genes in 3T3-L1 Adipocytes
}

\author{
Wajathip Bulanawichit, Thanagon Kirdin, and Tantip Boonsong* \\ Department of Biochemistry, Faculty of Medical Science, Naresuan University, Phitsanulok \\ 65000, Thailand \\ *Corresponding author.E-mail: tantips@hotmail.com \\ https://doi.org/10.12982/CMUJNS.2018.0007
}

\begin{abstract}
Obesity is a serious risk factor for metabolic diseases, such as atherosclerosis and type 2 diabetes mellitus. Obesity is caused by increasing adipose tissue mass via increasing cell numbers (hyperplasia) and adipocytes size (hypertrophy). Previous studies have found that natural bioactive compounds can be used to treat obesity. This study examined the anti-adipogenic mechanisms of brown rice (BR) and germinated brown rice (GBR) extracts (Khao Dawk Mali 105; KDML105 and Phitsanulok 2; PL2 cultivars) in 3T3-L1 adipocytes. The cytotoxicity effect of rice extracts was first measured by MTT assay and the non-toxic concentrations on triglyceride levels and expression of adipogenic, adipocytokine, and antioxidant genes were then investigated. The results showed that BR and GBR extracts at concentrations of $0.1,0.5$, and $1.0 \mathrm{mg} / \mathrm{ml}$ were not toxic. The triglyceride content in treated cells decreased significantly $(P<0.05)$ in a dose-dependent manner. The $m R N A$ expression

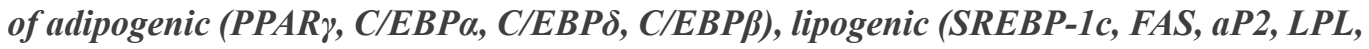
$A C C 1, A C C 2)$, and proinflammatory cytokine (TNFa, IL-6) genes decreased significantly $(P<0.05)$ in treated adipocytes as compared to the control, whereas lipolytic (ATGL, HSL), adiponectin, and antioxidant (SOD2, GPx4, CAT) genes increased significantly $(P<0.05)$. Furthermore, $m R N A$ expression of preadipocyte gene (Pref-1) was stable in adipocytes treated with GBR from KDML105 for 19 days throughout the differentiation, in contrast to the untreated cells. This study showed that by modulating adipogenic, adipocytokine, antioxidant, and preadipocyte gene expression, brown rice and germinated brown rice treatments of both PL2 and KDML105 cultivars may help reduce obesity.
\end{abstract}

Keywords: Obesity, Adipogenesis, Adipocytokine, Antioxidant, Rice, 3T3-L1 adipocytes 


\section{INTRODUCTION}

Excess fat accumulation caused by an imbalance of energy is a serious risk factor for obesity and other health problems, such as cardiovascular disease, hypertension, type 2 diabetes, dyslipidemia, and cancer (Gregor and Hotamisligil, 2007). Given these health risks, effective strategies to prevent and/or treat obesity are imperative.

The balance between adipocyte hyperplasia and hypertrophy strongly influences the metabolic outcome of obesity (Han et al., 2011). Smaller adipocyte cells retain insulin sensitivity and normal functioning (Tilg and Moschen, 2008), whereas large hypertrophic adipocytes cause insulin resistance (Naoto et al., 1999), modulate proinflammatory adipocytokine production (i.e., tumor necrosis factor (TNF)- $\alpha$; interleukin (IL)-6) (Paul and Wood, 2004), regulate oxidative stress (Zhao, 2016) and antioxidant molecules [i.e., superoxide dismutase (SOD); catalase (CAT); Glutathione peroxidase (GPx)] (Sfar et al., 2013; Rupérez et al., 2014), and decrease anti-inflammatory cytokine and adiponectin (Teng et al., 2014).

Adipogenesis, or adipocyte differentiation, occurs in a sequential manner and is regulated by several genes (Gregoire et al., 1998; Moseti et al., 2016), such as adipogenic transcription factors (peroxisome proliferator-activated receptor gamma (PPAR $)$, CCAAT/ enhancer-binding proteins $(C / E B P S)$, and lipogenic genes (lipoprotein lipase $(L P L)$, fatty acid synthase $(F A S)$, adipocyte protein $2(a P 2)$, sterol element binding protein-1 (SREBP-1c), and acetyl-CoA carboxylase $(A C C)$ ). In addition, adipogenesis is also influenced by preadipocyte genes, such as preadipocyte factor 1 (Pref-1) and SRY box 9 (SOX9) (Hudak and Sul, 2013).

Rice is a staple food, consumed daily by one-third of the world's population. Brown rice (BR) is a highly nutritious food that is relatively low in calories, high in fiber, gluten-free, and can be incorporated into a wide variety of dishes (Henry et al., 2009). BR and germinated brown rice (GBR) have more nutrients than white or polished rice (Huihui et al., 2014), including more fiber, minerals, phenolic acids, flavonoids, $\gamma$-aminobutyric acid (GABA), and $\gamma$-oryzanol (Patil and Khan, 2011; Karladee and Suriyong, 2012). Recent studies have shown beneficial health effects of BR and GBR; they improve glucose uptake (Chisayo et al., 2013), inhibit adipocyte differentiation (Ho et al., 2012), and increase lipolysis (Lim et al., 2014). In addition, studies have also investigated the molecular mechanisms of BR- and GBR-mediated adipogenesis, lipogenesis, lipolysis, inflammation, and oxidative stress (Ho et al., 2013; Imam and Ismail, 2015).

The bioactive compounds of BR and GBR extracts of Thai rice cultivars, including GABA, $\gamma$-oryzanol, phytic acid, and $\alpha$-tocopherol, were previously identified and analyzed (Jannoey et al., 2010a; Jannoey et al., 2010b; Moongngarm and Saetung, 2010). However, the biochemical mechanisms underlying their anti-obesity effects have not been studied. This study aimed to investigate the anti-obesity effects of BR and GBR extracts of Thai rice cultivars [O. sativa L. variety Phitsanulok 2 (PL2) and Khao Dawk Mali 105 (KDML105)] using 3T3$\mathrm{L} 1$ adipocytes as a model. The effects on adipocyte triglyceride levels and expression of adipogenic and other related genes, including preadipocyte, adipocytokine, and antioxidant genes, were all examined. 


\section{MATERIALS AND METHODS}

\section{Materials}

Dulbecco's modified Eagle's medium (DMEM), fetal bovine serum (FBS), and antibiotic-antifungal agents were purchased from Gibco BRL (Gaithersburg, MD, USA). 3-isobutyl-1-methylxanthine (IBMX), dexamethasone (DEX), insulin, caffeine, and GABA were bought from Sigma Chemicals (Sigma-Aldrich, USA). All other chemicals used were high quality and commercially available.

\section{Sample preparation}

BR (PL2 and KDML105) was obtained from the Phitsanulok Rice Research Center and Lopburi Rice Research Center, Thailand. The germination method of BR was modified from Patil and Khan (2011). Briefly, $100 \mathrm{~g}$ of BR was soaked in $200 \mathrm{ml}$ of sterile water at room temperature for $6 \mathrm{hrs}$ and germinated for $24 \mathrm{hrs}$. GBR grains were sampled after $24 \mathrm{hrs}$ of germination. The extraction procedure was modified from a previous study (Ho et al., 2013). First, $50 \mathrm{~g}$ of BR and GBR powder was placed in a 250 -ml flask containing $100 \mathrm{ml}$ of $70 \%$ $(\mathrm{v} / \mathrm{v})$ methanol solution. The mixture was shaken $(180 \mathrm{rpm})$ for $20 \mathrm{hrs}$ at $37^{\circ} \mathrm{C}$. The supernatant was collected in a $250-\mathrm{ml}$ flask. The same volume of $70 \%$ methanol solution was added to the pellet as described above, and the extraction was repeated. The collected supernatant (150 ml) was filtered through 1-micron filter paper (Whatman, USA) and evaporated by rotary evaporation (BUCHI, Switzerland). The crude rice extracts were weighed and dissolved in dimethyl sulfoxide (DMSO) (final concentration $0.1 \%$ ) as the stock solution.

\section{T3-L1 cell culture}

3T3-L1 preadipocytes (\#CL-173, ATCC) were grown in DMEM containing 10\% FBS and $1 \%$ antibiotic-antifungal agent (Basal medium; $\mathrm{BM}$ ) at $37^{\circ} \mathrm{C}$ in a $5 \% \mathrm{CO}_{2}$ incubator. After two days post-confluent, cells (day 0) were differentiated for three days in differentiation medium 1 (DM1) [BM, 1 mM IBMX, $2 \mu \mathrm{M}$ DEX] (day 3) and for two days in differentiation medium 2 (DM2) [BM, $1 \mu \mathrm{g} / \mathrm{ml}$ insulin] (day 5). After cell differentiation finished, the cells were maintained and matured in BM, which was replaced with fresh BM on day 7 and 9.

\section{Cell viability}

To determine cell viability, 3T3-L1 cells were incubated with BR and GBR extracts at various concentrations $(0,0.1,0.5,1.0 \mathrm{mg} / \mathrm{ml})$ for $24 \mathrm{hrs}$. The control cells received the same amount of DMSO $(0.1 \% \mathrm{v} / \mathrm{v})$. After incubation, the medium was replaced with serum-free medium containing $20 \mu \mathrm{l}$ of 3-(4, 5-Dimethylthiazol-2-yl)-2, 5-diphenyltetrazolium bromide (MTT) $(5 \mathrm{mg} / \mathrm{ml})$ and incubated for $2 \mathrm{hrs}$ at $37^{\circ} \mathrm{C}$. The solution was discarded, and $100 \mu \mathrm{l}$ of DMSO was added to each well. The absorbance was measured at $595 \mathrm{~nm}$ using a microplate reader (Labsystems, Finland).

\section{Triglyceride quantification}

To examine triglyceride levels, cells were treated with BR and GBR extracts at concentrations of $0.1,0.5$ and $1.0 \mathrm{mg} / \mathrm{ml}$, starting from the initiation of differentiation until day 9, as described above. The triglyceride contents were estimated by a commercial triglyceride assay kit (Triglycerides liquicolor ${ }^{\text {mono }}$, Germany) as compared to untreated cells. GABA (1 $\mathrm{mg} / \mathrm{ml})$ and caffeine $(0.1 \mathrm{mg} / \mathrm{ml})$ were used as the positive controls. Briefly, the adipocytes were lysed in lysis buffer (Tris-HCL $25 \mathrm{mM}$, EDTA $1 \mathrm{mM}, \mathrm{pH}$ 7.4) by using a sonicator 
(Evan et al., 2000) and the triglycerides were then hydrolyzed with lipases. The product, quinonemine, formed from hydrogen peroxide, 4-aminoantipyrine, and 4-chlorophenol under the catalytic influence of peroxidase, was measured by absorbance at $500 \mathrm{~nm}$. The amount of total protein was calculated with a BCA protein assay kit (Thermo, USA) and normalized to triglyceride contents.

\section{mRNA expression measurement by quantitative real-time PCR (qPCR)}

After incubating the cells for nine days (from the start of differentiation) in BR and GBR extracts, total RNA was extracted from 3T3-L1 adipocytes using Ribozol reagent, according to the manufacturer's instructions (AMRESCO, USA). RNA samples ( $1 \mu \mathrm{g})$ were reverse-transcribed to the complementary DNA using the random primer and MMLV reverse transcriptase (Promega, Madison, WI, USA). The mRNA expression was measured by qPCR using SYBR Green (RBC Bioscience, Taiwan). The primer sequences are shown in Table 1. The reaction mixtures were incubated for an initial denaturation at $95^{\circ} \mathrm{C}$ for $10 \mathrm{~min}$, followed by 40 PCR cycles. Each cycle was performed at $95^{\circ} \mathrm{C}$ for 30 seconds, $58^{\circ} \mathrm{C}$ for 30 seconds, and $72^{\circ} \mathrm{C}$ for 30 seconds (ESCO Swift ${ }^{\mathrm{TM}}$ Real-Time PCR Thermal Cyclers, Singapore). The comparative $\mathrm{C}_{\mathrm{T}}$ method $\left(2^{-\Delta \Delta \mathrm{CT}}\right)$ was used to measure relative mRNA expression, and the expression was normalized to $\beta$-actin. The expression of each different mRNA in the untreated cells was designated as 1 , and the relative levels of transcripts in treated samples were expressed as the fold change. Two independent experiments were performed, each in triplicate.

\section{GABA and $\gamma$-oryzanol determination}

The GABA and $\gamma$-oryzanol contents in the BR and GBR extracts were measured by the Central Laboratory (Thailand) Co., Ltd. The GABA content was measured by Gas Chromatography (GC) (Agilent Technologies 6890 N; column: Phenomenex USA Zebron $10 \mathrm{~m} \times 0.25 \mathrm{~mm} \times 0.25 \mathrm{~m}$ ) - Mass Selective Detector (MSD) (Agilent Technologies 5973 inert USA) method as described by Herbert et al. (2000) and Iwaki and Kitada (2007). The $\gamma$-oryzanol content was determined by High Performance Liquid Chromatography (HPLC) technique based on Wihong et al. (2014).

Table 1. Primer sequences used in qPCR.

\begin{tabular}{lll}
\hline Gene & \multicolumn{1}{c}{ Forward primer (5'-3') } & \multicolumn{1}{c}{ Reverse primer (5'-3') } \\
\hline$\beta$-actin & ATTTATCCCGGTGTACTGTG & CGGAAGTCAGAGAGGCGTAGTAG \\
$P r e f-1$ & GCGCCAACAATGGAACTTGCGT & GAGGGGTACTCTTGTTGAGCTC \\
$P P A R \gamma$ & GCGGCCATGGAGTCAATG & CGCTGATGCACTGCCTATGA \\
$C / E B P \alpha$ & AATGGCAGTGTGCACGTCTA & CCCCAGCCGTTAGTGAAGAG \\
$C / E B P \beta$ & GGGGTTGTTGATGTTTTTGG & CGAAACGGAAAAGGTTCTCA \\
$C / E B P \delta$ & TCCACGACTCCTGCCATGTA & GCGGCCATGGAGTCAATG \\
$S R E B P-1 c$ & CTGCAGACCCTGGTGAGTG & GACCGGTAGCGCTTCTCAAT \\
$F A S$ & TTTGTAAACGTCCTCACCCGA & CGGAAGTTCAGAGAGGCGTAGTAG \\
$a P 2$ & CATGGCCAAGCCCAACAT & CGCCCAGTTTGAAGGAAATC \\
LPL & ACTTGTCATCTCATTCCTGG & TCTCATACATTCCCGTTACC \\
Adiponectin & CAGGTCTTCTTGGTCCTAAGGG & GTCCACATTCTTTTCCTGATACTG \\
\hline
\end{tabular}


Table 1. Continued.

\begin{tabular}{lll}
\hline Gene & \multicolumn{1}{c}{ Forward primer (5'-3') } & \multicolumn{1}{c}{ Reverse primer (5'-3') } \\
\hline$A T G L$ & ATTTATCCCGGTGTACTGTG & GGGACACTGTGATGGTATTC \\
$H S L$ & ACTCAGACCAGAAGGCACTA & TAGTTCCAGGAAGGAGTTGA \\
$A C C 1$ & TGAGGAGGACCGCATTTATC & GAAGCTTCCTTCGTGACCAG \\
$A C C 2$ & ACAGAGATTTCACCGTTGCGT & CGCAGCGATGCCATTGT \\
$T N F-\alpha$ & GATTTGCTATCTCATACCAGGAGAA & AAGTCTAAGTACTTGGGCAGATTGA \\
$I L-6$ & AGGCTTAATTACACATGTTCTCTGG & TTATATCCAGTTTGGTAGCATCCAT \\
$S O D 2$ & AACCCAAAGGAGAGTGCTG & CAGCAATCTGTTAAGCGACCT \\
$G P x 4$ & CCAATAAGAGACGTCGTGGG & GGCTTAAGTAAGCGGCTCAG \\
$C A T$ & AGCCCTGACAAAATGCTTCA & GCTGAAGCTGTTGGGGTAAT \\
\hline
\end{tabular}

\section{Statistical analysis}

Values were expressed as mean \pm standard deviation (S.D.). One-way analysis of variance (ANOVA) and $t$-test were used for multiple comparisons. Treatment effects were analyzed using Tukey's multiple range tests by SPSS 17.0 software. Differences were considered significant with $P<0.05$.

\section{RESULTS}

\section{Cell viability}

BR and GBR extracts from both cultivars at various concentrations $(0.1,0.5$, and 1.0 $\mathrm{mg} / \mathrm{ml}$ ) showed no cytotoxicity effect on 3T3-L1 cells (Figure 1).

(A)

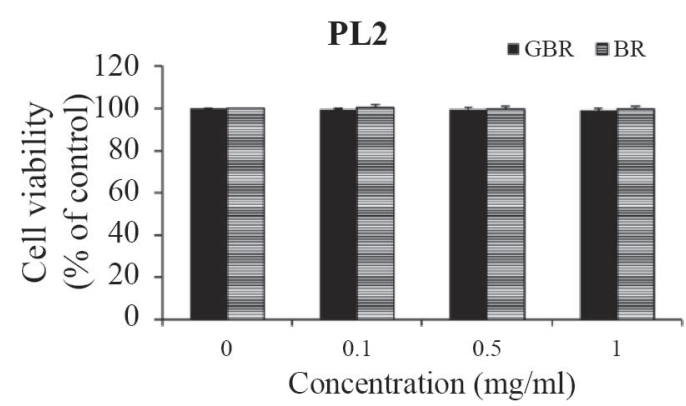

(B)

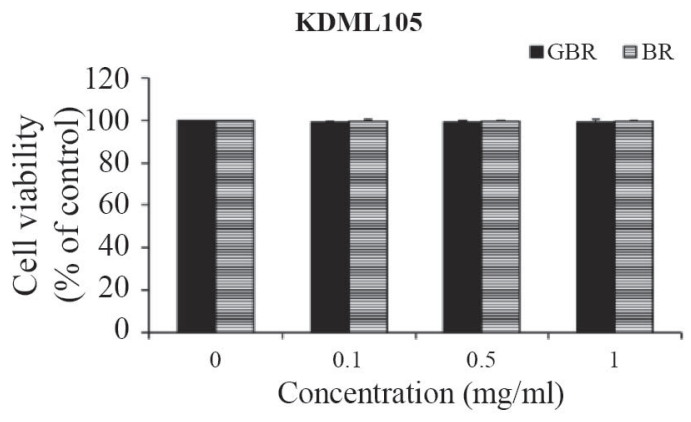

Figure 1. Effect of BR and GBR extracts $(0.1,0.5$, and $1.0 \mathrm{mg} / \mathrm{ml})$ from PL2 (A) and KDML105 (B) cultivars on viability of 3T3-L1 cells.

\section{Triglyceride levels}

$\mathrm{BR}$ and GBR extracts from both rice cultivars significantly decreased $(P<0.05)$ intracellular triglyceride levels in 3T3-L1 adipocytes (Figure 2A and 2B) in a dose-dependent manner. Figure $2 \mathrm{C}$ shows the results of the positive controls, caffeine $(0.1 \mathrm{mg} / \mathrm{ml})$ and GABA $(1 \mathrm{mg} / \mathrm{ml})$; both significantly reduced triglyceride contents in adipocytes compared to the control group $(P<0.05)$. 
(A)

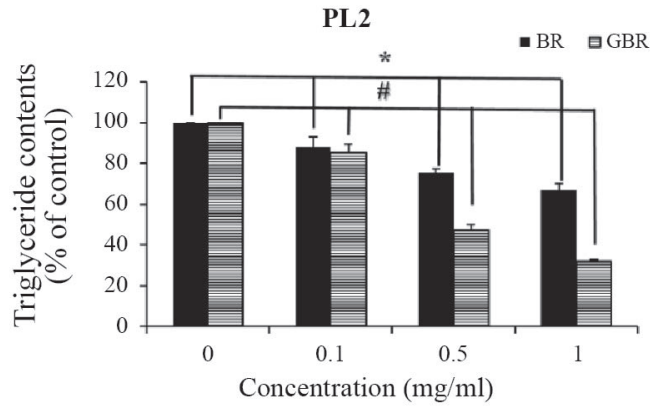

(B)

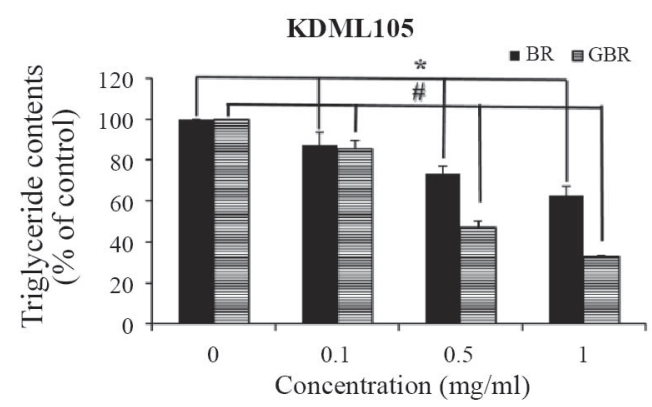

(C)

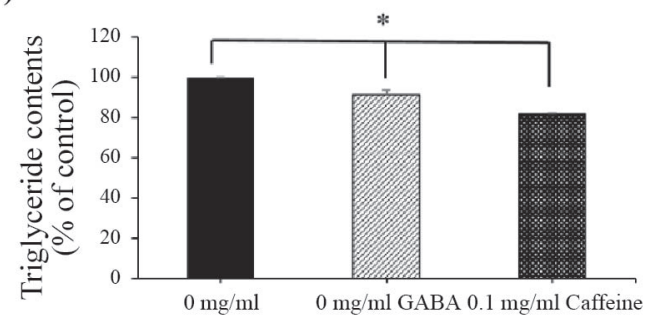

Figure 2. Effect of BR and GBR extracts on triglyceride contents in 3T3-L1 adipocytes. Cells were treated with rice extracts from PL2 (A), KDML105 (B) cultivars $(0.1,0.5$, and $1.0 \mathrm{mg} / \mathrm{ml}), \mathrm{GABA}(1.0 \mathrm{mg} / \mathrm{ml})$, and caffeine $(0.1 \mathrm{mg} / \mathrm{ml})(C)$ for nine days. Triglyceride levels were determined by Triglyceride kit assay.

Note: $* P<0.05, \# P<0.05$ compared to control of $\mathrm{BR}$ and GBR treatments, respectively.

\section{GABA and $\gamma$-oryzanol contents}

In both cultivars, GBR contained more GABA than BR, while GBR contained less $\gamma$-oryzanol than BR (Table 2).

Table 2. The GABA and $\gamma$-oryzanol contents in BR and GBR extracts in PL2 and KDML105 cultivars.

\begin{tabular}{lcc}
\hline Samples & GABA (mg/100g) & $\boldsymbol{\gamma}$-oryzanol (mg/100g) \\
\hline BR (PL2) & $<1$ & 34.88 \\
BR (KDML105) & $<1$ & 40.97 \\
GBR (PL2) & 16.46 & 31.55 \\
GBR (KDML105) & 36.88 & 28.65 \\
\hline
\end{tabular}

Note: Analyzed by the Central Laboratory (Thailand) Co., Ltd.

mRNA expression of adipogenic, lipogenic, and lipolytic genes

The results examined by qPCR showed that the mRNA expression of adipogenic transcription factors $(P P A R \gamma, C / E B P \alpha, C / E B P \beta$, and $C / E B P \delta)$ in 3T3-L1 cells treated with BR and GBR from PL2 (Figure 3A) and KDML105 (Figure 3B) cultivars decreased significantly compared with the control $(P<0.05)$. The treatments also suppressed the expression of lipogenic genes (SREBP-1c, FAS, LPL, aP2, ACC1, and ACC2) (Figure 3). However, the expression of lipolytic genes $A T G L$ and $H S L$ increased significantly in treated cells as compared to untreated control cells $(P<0.05)$ (Figure 4). 
(A) PL2
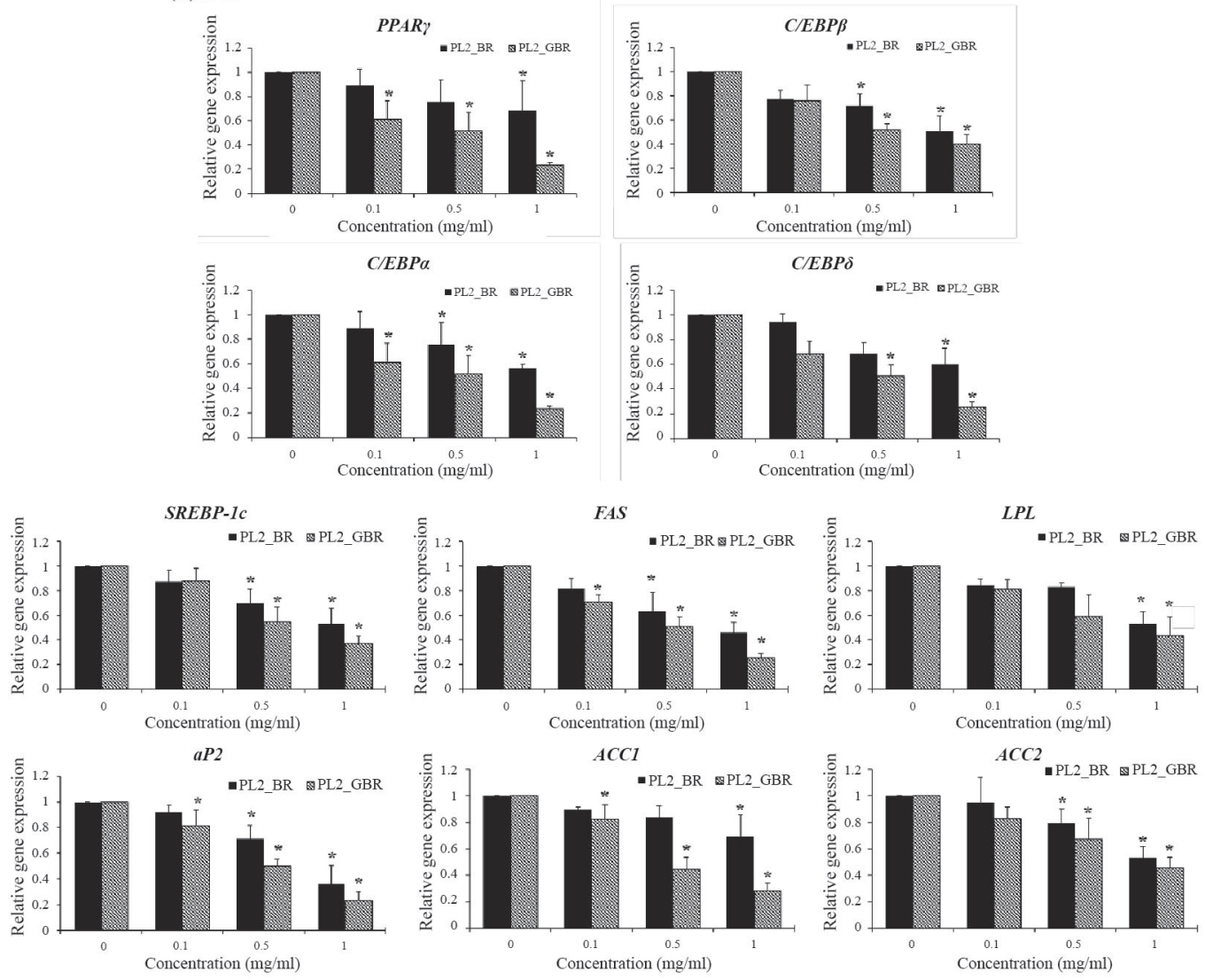

Figure 3. Effect of BR and GBR extracts from PL2 (A) and KDML105 (B) cultivars on adipogenic and lipogenic gene expression by qPCR.

Note: ${ }^{*} P<0.05$ compared to control. 


\section{(B) KDML105}
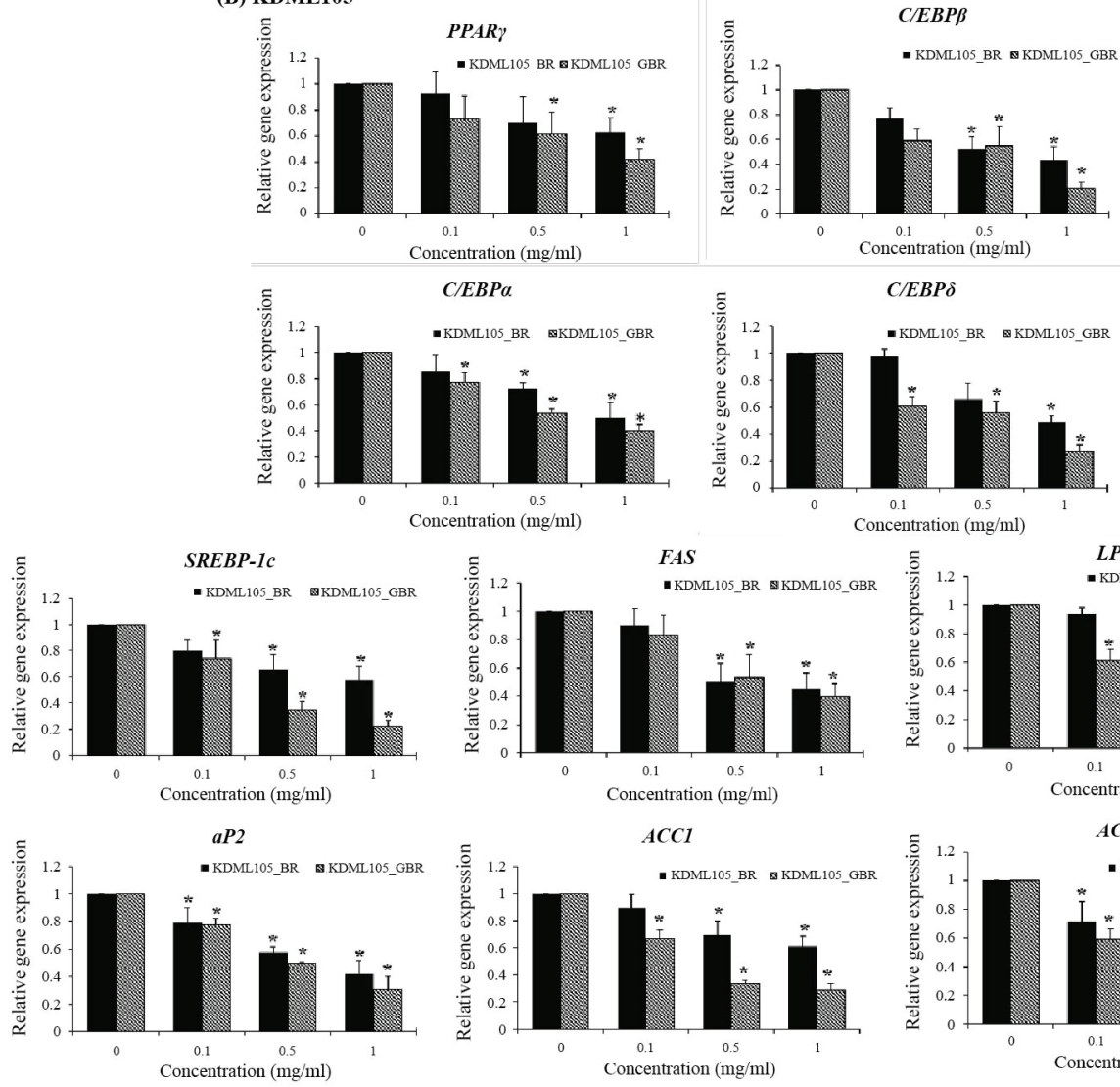

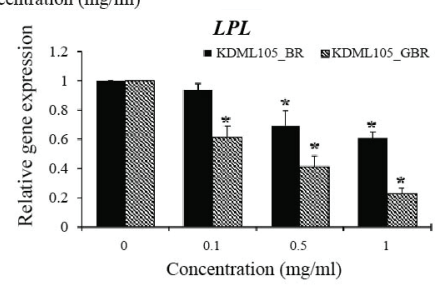

ACC2

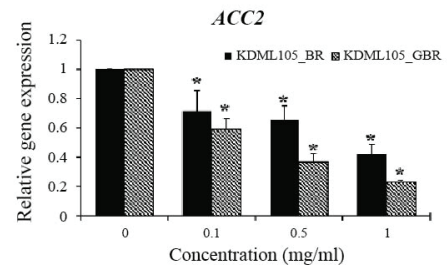

Figure 3. Continued.

Note: ${ }^{*} P<0.05$ compared to control. 
(A)
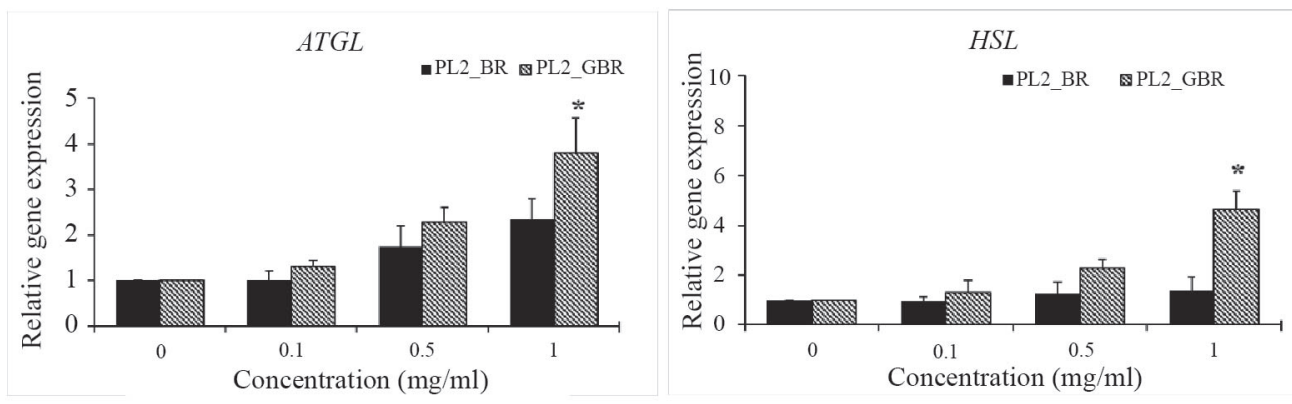

(B)
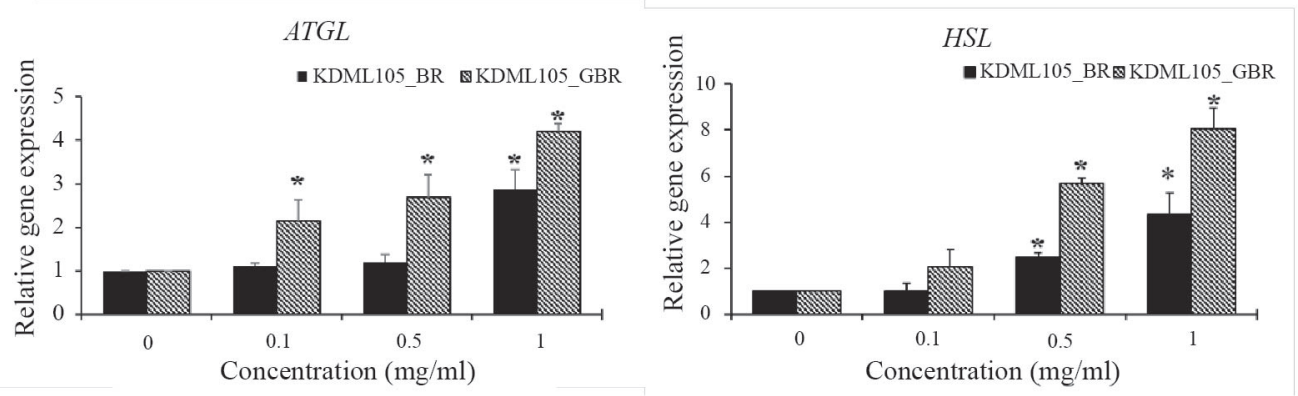

Figure 4. Effect of BR and GBR extracts from PL2 (A) and KDML105 (B) cultivars on lipolytic gene expression by qPCR.

Note: $* P<0.05$ compared to control.

\section{mRNA expression of adipocytokines}

BR and GBR extracts significantly decreased mRNA expression of proinflammatory cytokines $(T N F-\alpha$ and $I L-6)$ in mature adipocytes $(P<0.05)$ (Figures $5 \mathrm{~A}$ and $5 \mathrm{~B})$. In addition, adiponectin mRNA expression was significantly up-regulated $(P<0.05)$ (Figure 5C).

\section{mRNA expression of antioxidant genes}

The expression of antioxidant genes (SOD2, GPX4 and CAT) increased significantly $(P<0.05)$ in adipocytes treated with BR and GBR extracts of both PL2 and KDML105 cultivars (Figure 6). 
(A)

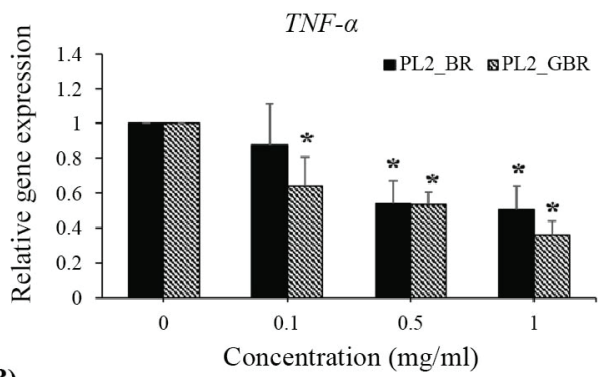

(B)

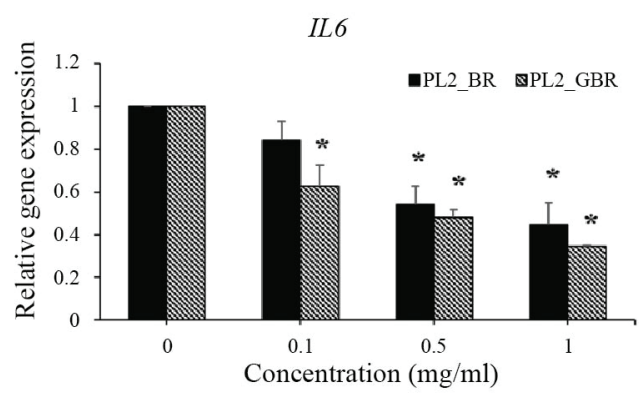

(C)

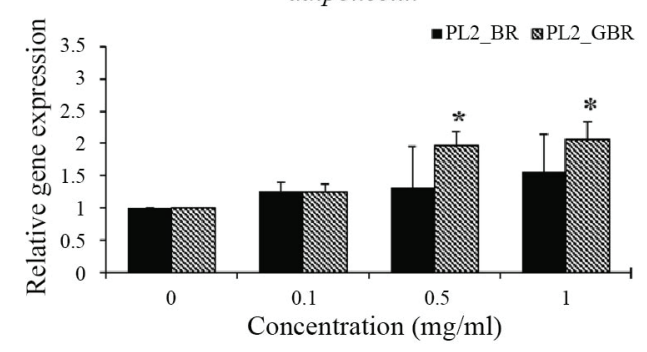

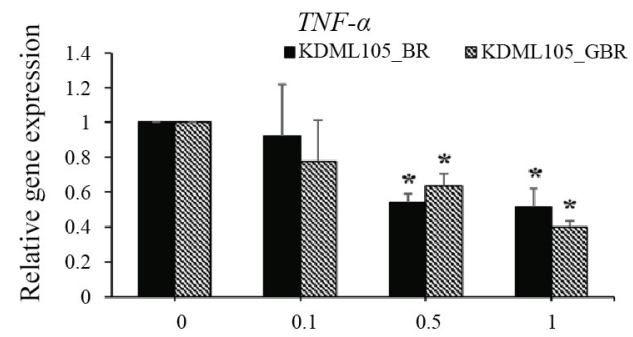

Concentration (mg/ml)

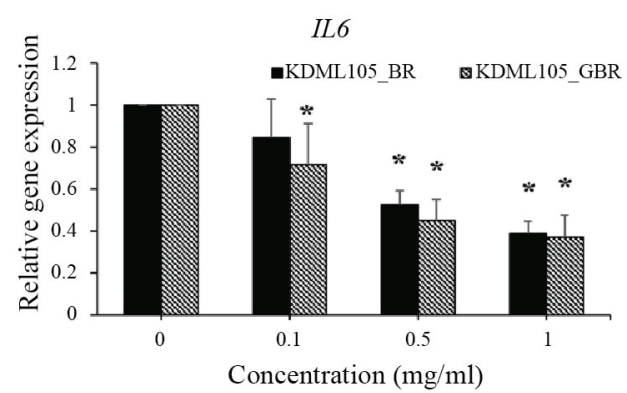

adiponectin

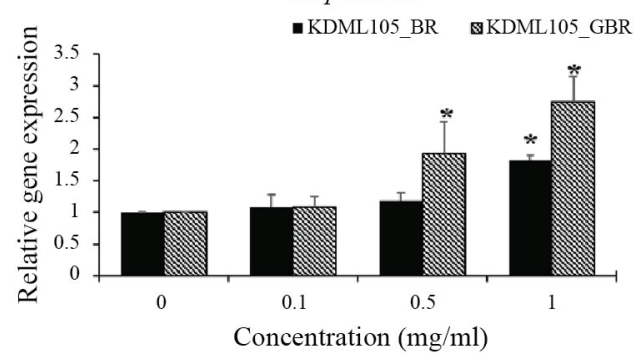

Figure 5. Effect of BR and GBR extracts from PL2 and KDML105 cultivars on TNF- $\alpha$ (A) and $I L-6$ (B), and adiponectin (C) gene expression by qPCR.

Note: $* P<0.05$ compared to control. 
(A) PL2

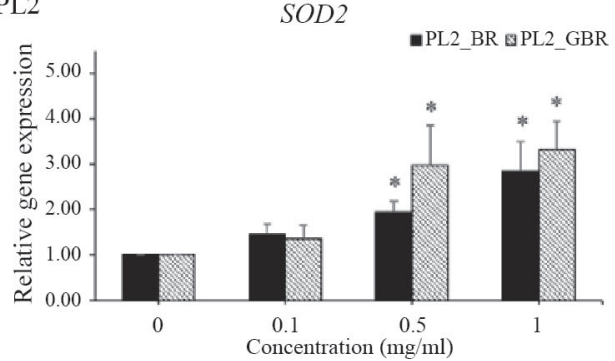

CAT

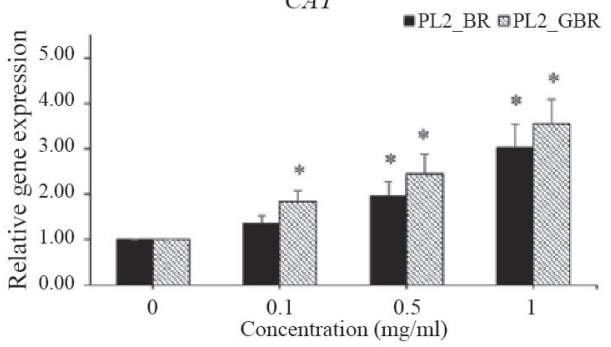

GPx4

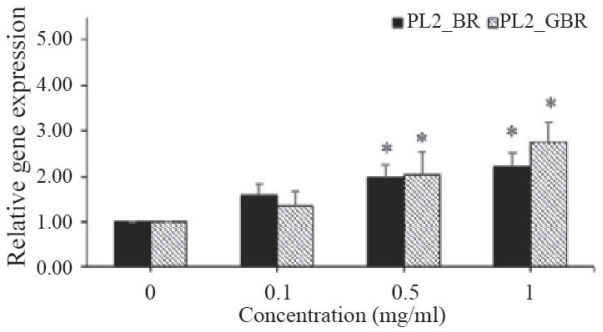

(B) KDML105

SOD2

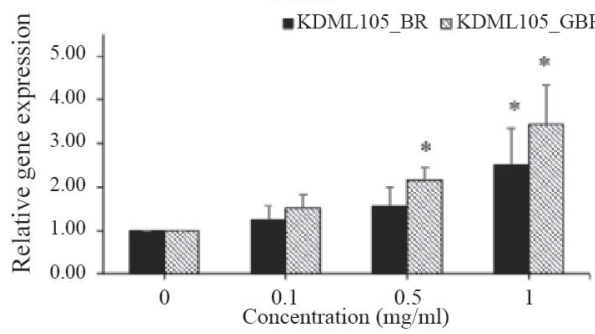

CAT

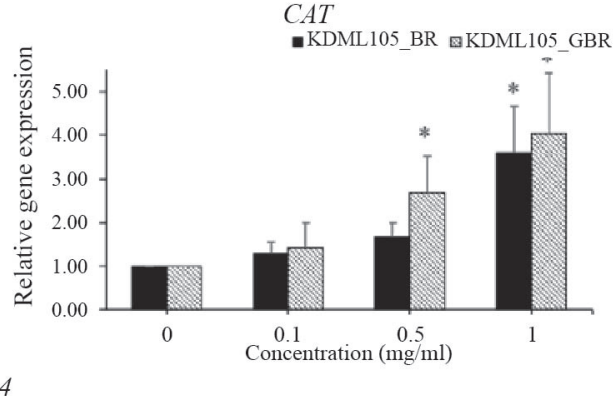

- KDML105 BR $₫$ KDML105 GBR

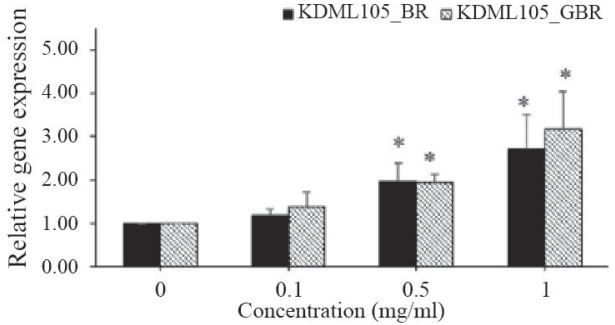

Figure 6. Effect of BR and GBR extracts from PL2 (A) and KDML105 (B) cultivars on SOD2, GPx4, and CAT gene expression by qPCR.

Note: $* P<0.05$ compared to control. 


\section{mRNA expression of a preadipocyte gene}

The expression of Pref-1, a preadipocyte gene, in untreated 3T3-L1 adipocytes was gradually down-regulated during adipocyte differentiation (0-19 days) from day 7 (Figure 7). However, when treated with $1 \mathrm{mg} / \mathrm{ml} \mathrm{GBR}$ of KDML105 cultivar, its expression increased significantly $(P<0.05)$ from day 13 until differentiation ended (Figure 7).

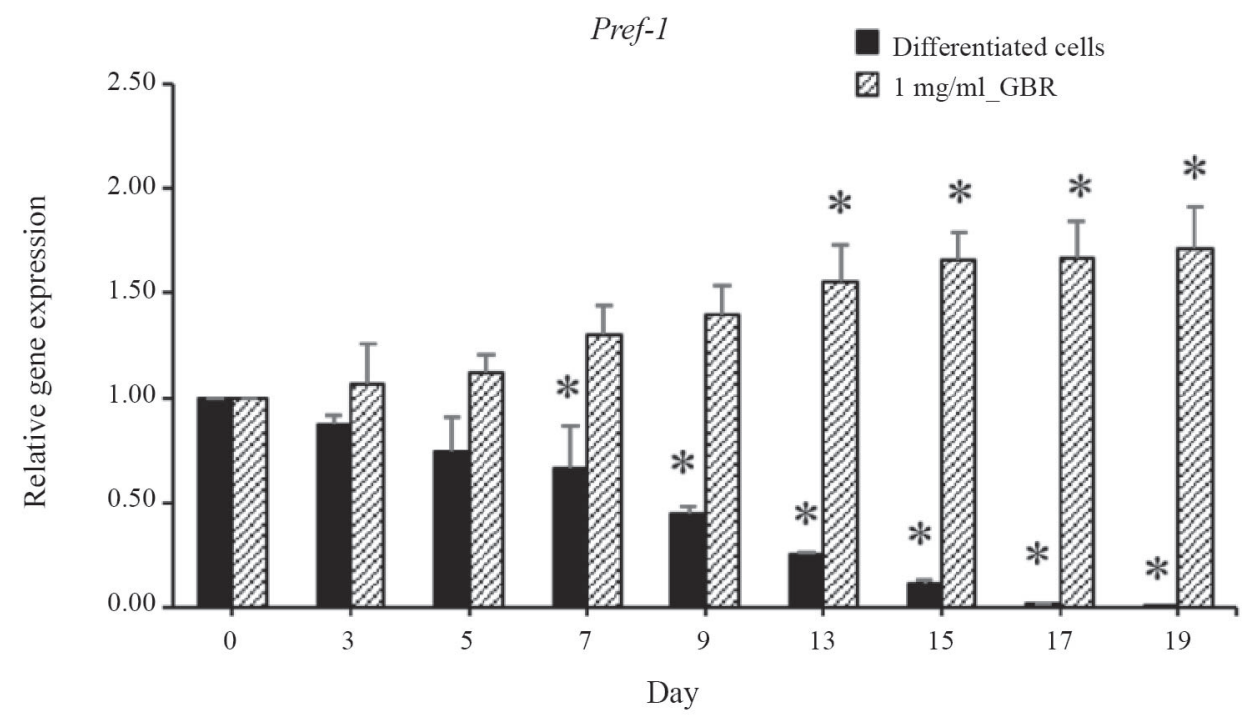

Figure 7. Effect of GBR extract from KDML105 cultivar on Pref-1 gene expression.

Note: $* P<0.05$ compared to differentiated cells.

\section{DISCUSSION}

This study examined the effects of brown rice and germinated brown rice extracts of PL2 and KDML105 Thai rice cultivars on adipocyte differentiation. These effects were in the absence of any influence on cell viability as tested by MTT assay. We also investigated the molecular mechanisms of BR and GBR extracts on adipocyte differentiation through analyzing mRNA expression of genes known to be associated with adipogenesis, lipogenesis, lipolysis, and inflammation, as well as oxidative status by quantitative real-time PCR.

The results showed that mRNA levels of adipogenic transcription factors $C / E B P \alpha, C /$ $E B P \beta, C / E B P \delta$, and $P P A R \gamma$ were significantly down-regulated in a dose-dependent manner in adipocytes treated with both BR and GBR extracts. These adipogenic transcription factors can regulate each other (Evan et al., 2011) and control the expression of lipogenic genes, such as SREBP-1c, FAS, ACC, $a P 2$, and LPL (Wu et al., 1996; Lee et al., 2007), which has been implicated in the differentiation of preadipocytes to mature adipocytes. As expected, mRNA expression of lipogenic genes, including SREBP-1c, FAS, ACC1, ACC2, aP2, and LPL, were suppressed in a dose-dependent manner $(P<0.05)$ in adipocytes treated with BR and GBR extracts. We also showed that BR and, to a greater extent, GBR significantly activated mRNA expression of the lipolytic genes $A T G L$ and $H S L$; this can reduce lipids in 3T3-L1 adipocytes.

Adipocytes produce several adipocytokines, including leptin, visfatin, resistin, adiponectin, and proinflammatory cytokines, such as TNF- $\alpha$ and IL6 (De Silva et al., 2011). 
Paul and Wood (2004) reported that TNF- $\alpha$ and IL6 have inhibitory effects on insulin signaling pathways, causing insulin resistance. Previous studies found that TNF- $\alpha$ activated IL6 production (Chiellini et al., 2002) and inhibited the synthesis of anti-inflammatory cytokines, such as adiponectin and visfatin (Fernández-Sánchez et al., 2011). Therefore, decreasing proinflammatory and/or increasing anti-inflammatory cytokines may improve insulin sensitivity. Wang et al. (2010) showed that treating type 2 diabetic rats with BR and GBR increased adiponectin and reduced blood sugar levels. In this study, mRNA expression of both $T N F-\alpha$ and IL6 decreased, while adiponectin increased in cells treated with BR and GBR extracts of both rice cultivars. This could potentially improve insulin sensitivity, although this needs further study.

BR and GBR contain several antioxidants, including $\gamma$-oryzanol, phytic acid, ferulic acid, phenolics, and flavonoids, that might have effective antioxidant effects (Kaukovirta et al., 2004; Patil and Khan, 2011). A recent study demonstrated that BR and GBR extracts of Malaysian local rice increased the activity of antioxidant enzymes (SOD and GPx) in rabbits (Mohd Esa et al., 2013). In agreement with our study, BR and GBR extracts can up-regulate mRNA levels of SOD2, GPX4, and CAT in 3T3-L1 adipocytes.

In addition to adipocyte genes, we also examined the effect of BR and GBR extracts on a preadipocyte gene, Pref-1. Pref-1 is a novel member of the epidermal growth factor (EGF)-like family of proteins (Hudak and Sul, 2013); it is a marker for preadipocyte. It is believed to negatively regulate adipocyte differentiation through MEK-ERK and SOX9 signaling pathways, leading to inhibition of adipogenesis (Wang et al., 2010). Thus, the temporary up-regulation of Pref-1 expression might play an imperative role in adipogenesis (Jing et al., 2009). Our results showed that the basal expression of Pref-1 was abundant in preadipocytes and down-regulated at day 7. However, when cells were treated with GBR extracts of KDML105 cultivar from the start of differentiation, Pref- 1 mRNA expression was stable throughout differentiation. These results suggest that restoring Pref- 1 mRNA expression by GBR extracts may affect adipocyte differentiation, although this needs further study.

We extracted BR and GBR using a methanol-based method described by Ho et al. (2012) to generate rice grain extracts with significant amounts of bioactive compounds, including GABA, phenolics, and $\gamma$-oryzanol. We demonstrated that both BR and GBR methanol extractions reduced lipid accumulation in 3T3-L1 adipocytes, in agreement with Ho et al. (2012). Ho et al. (2012) speculated that bioactive compounds, such as GABA, vitamin E, and $\gamma$-oryzanol, played a role in improving lipid metabolism, although they were not able to clearly identify the bioactive components in the extraction. We analyzed $70 \%$-methanol extractions from BR and GBR (Table 2) and found that GBR had more GABA than BR, but less $\gamma$-oryzanol; this was inconsistent with Imam et al. (2012). This may be due to the sample preparation during germination and/or dehusking.

Nicolaysen et al. (2007) found GABA receptors in the adipocytes and Ohara et al. (2011) suggested these may modulate adiponectin levels via vagal systems. We used GABA as a positive control and it significantly decreased triglyceride contents (Figure 2), although we do not fully understand its mechanism of action.

$\gamma$-oryzanol has been shown to directly enhance adiponectin secretion in adipocytes, partly via suppressing NF- $\mathrm{B}$ activation (Nagasaka et al., 2007; Ohara et al., 2009). Minatel et al. (2016) studied the anti-obesity effect of $\gamma$-oryzanol and showed that $\gamma$-oryzanol down- 
regulated the expression of adipogenic transcription factors, such as $P P A R \gamma$ and $C / E B P s$; this inhibited mature adipocyte differentiation. In addition, Ho et al. (2013) speculated that $\gamma$-oryzanol, which remarkably increased in GBR during germination, has anti-adipogenic effects by decreasing the mRNA expression of FAS. These results suggest that GABA and $\gamma$-oryzanol may play a vital role in adipogenesis. Other bioactive compounds present in the rice extracts, such as $\alpha$-tocopherol, kaempferol, vanillin, fisetin, ferulic acid, and phytic acid (Partil and Khan, 2011; Imam et al., 2016), may synergistically potentiate the effects of GABA and $\gamma$-oryzanol.

We propose that the molecular mechanism of BR- and GBR-mediated effects on adipogenic, adipocytokine, and antioxidant-related genes in 3T3-L1 adipocytes may occur via multiple pathways (Figure 8), including regulating adipocyte differentiation, adipocytokine production, and oxidative status. The modulation of adipogenic, adipocytokine, antioxidant, and preadipocyte gene expression by BR and GBR treatments of both PL2 and KDML105 cultivars may help reduce obesity.

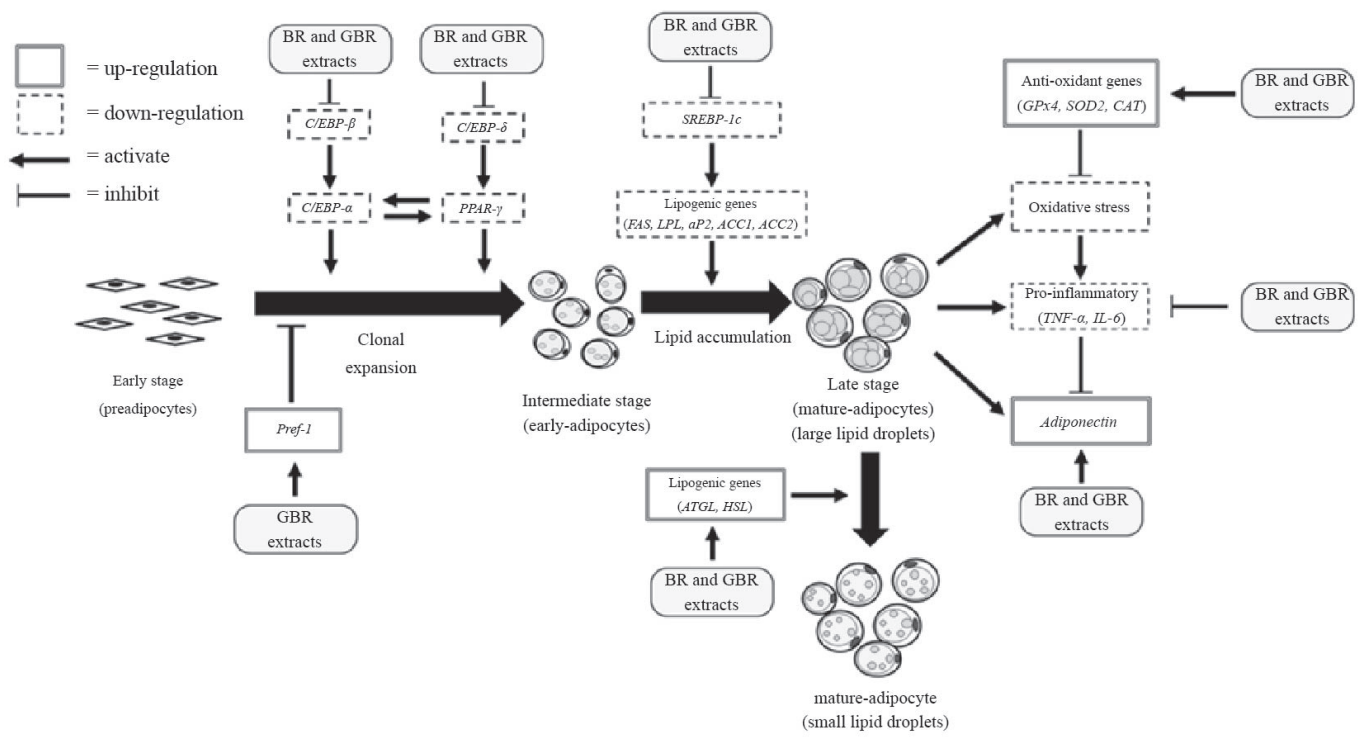

Figure 8. Schematic diagram showing proposed targets of brown rice (BR) and germinated brown rice (GBR) extracts in 3T3-L1 adipocyte differentiation.

Note: Pref-1: preadipocyte factor 1; C/EBP- $\alpha$ : CCAAT/enhancer binding protein alpha; $C / E B P-\beta$ : CCAAT/enhancer binding protein beta; $C / E B P-\delta$ : CCAAT/enhancer binding protein delta; PPAR : peroxisome proliferation-activated receptor gamma; SREBP-1c: sterol regulatory element binding protein $1 \mathrm{c}$; $F A S$ : fatty acid synthase; $A C C$ : acetyl coA carboxylase; $L P L$ : lipoprotein lipase; $A T G L$ : adipocyte triglyceride lipase; $H S L$ : hormone sensitive lipase; GPx4:glutathione peroxidase; SOD2: superoxide dismutase 2; CAT: catalase; $T N F-\alpha$ : tumor necrosis factor alpha; $I L-6$ : interleukin 6. 


\section{CONCLUSION}

This study demonstrated that BR and GBR extracts of PL2 and KDML105 Thai rice cultivars disturbed 3T3-L1 adipocyte differentiation by reducing lipid contents through downregulating adipogenic and lipogenic gene expression, as well as up-regulating the expression of lipolytic and antioxidant genes. In addition, they activated expression of adiponectin and inhibited proinflammatory cytokine genes. Thus, BR and GBR extracts may help reduce obesity.

\section{ACKNOWLEDGEMENTS}

We gratefully thank the National Research Council of Thailand for their financial support (code R2559B061). In addition we thank the Phitsanulok Rice Seed Center and Lopburi Rice Seed Center for providing the rice grains (Phitsanulok 2 and Khao Dawk Mali 105) and the Department of Biochemistry, Faculty of Medical Science, Naresuan University for use of its facilities. We appreciate Panatda Jannoey suggestions for rice germination. Finally, we thank Roy Morien, Naresuan University Language Centre, and Gavin Paul Reynolds, Faculty of Medical Science for proofreading and editing this manuscript.

\section{REFERENCES}

Chiellini, C., Bertacca, A., Novelli, S.E., Gorgun, C.Z., Ciccarone, A., Giordano, A., Xu, H., Soukas, A., Costa, M., Gandini, D., et al. 2002. Obesity modulates the expression of haptoglobin in the white adipose tissue via TNF alpha. Journal of Physiology. 190(2): 251-258. https://doi.org/10.1002/jcp.10061

Chisayo, K., Kouichi, Y., Chitoshi, T., Masayuki, M., Michio, S., and Hiroaki, M. 2013. Natural food science based novel approach toward prevention and treatment of obesity and type 2 diabetes: Recent studies on brown rice and $\gamma$-oryzanol. Obesity Research and Clinical Practice. 7(1): 165-172. https://doi.org/10.1016/j.orcp.2013.02.003

De Silva, A.P. 2011. Evidence based guidelines for preparation before upper gastrointestinal endoscopy (UGIE), Gastrointestinal Endoscopy. InTech. https://doi.org/10.5772/21914

Evan, R.D., Hsu, C.H., Wang, X., Sakai, S., Freeman, M.W., Gonzalez, F.J., and Spiegelman, B.M. 2000. C/EBP $\alpha$ induces adipogenesis through PPAR $\gamma$ : a unified pathway. Gene and development. 16(1): 22-27. https://doi.org/10.1101/gad.948702

Fernández-Sánchez, A., Madrigal-Santillán, E., Bautista, M., Esquivel-Soto, J., MoralesGonzález, A., Esquivel-Chirino, C., Durante-Montiel, I., Sánchez-Rivera, G., ValadezVega, C., and Morales-González1, J.A. 2011. Inflammation, oxidative stress, and obesity. International Journal of Molecular Sciences. 12(5): 3117-3132. https://doi.org/ 10.3390/ijms12053117

Gregoire, F.M, Smas, C.M., and Sul, H.S. 1998. Understanding adipocyte differentiation. Physiological Reviews. 78(3): 783-809.

Gregor, M.F., and Hotamisligil, G.S. 2007. Thematic review series: Adipocyte biology. Adipocyte stress: The endoplasmic reticulum and metabolic disease. Journal of Lipid Research. 48:1905-1914. https://doi.org/10.1194/jlr.R700007-JLR200 
Han, J., Lee, J.E., Jin, J., Lim, J.S., Oh, N., Kim, K., Chang, S.I., Shibuya, M., Kim, H., and Koh, G.Y. 2011. The spatiotemporal development of adipose tissue. Journal of Development. 138: 5027-5037. https://doi.org/10.1242/dev.067686

Henry, C.J., Newens, K.J., and Lightowler, H.J. 2009. Low-glycaemic index sweetener-based beverages reduce 24-h glucose profiles in healthy adults. Journal Human Nutrition Dietetics. 22(1): 77-80. https://doi.org/10.1111/j.1365-277X.2008.00930

Herbert, P., Barros, P., Ratola, N., and Alves, A. 2000. HPCL determination of amino acid in musts and port wine using OPA/FMOC derivatives. Food Chemistry and Toxicology. 65(7): 1130-1133. https://doi.org/10.1111/j.1365-2621.2000.tb10251.x

Ho, J.N., Son, M.E., Lim, W.C., Lim, S.T., and Cho, H.Y. 2012. Anti-obesity effects of germinated brown rice extract through down-regulation of lipogenic genes in high fat diet-induced obese mice. Bioscience, Biotechnology, and Biochemistry. 76(6): 10681074. https://doi.org/10.1271/bbb.110666

Ho, J.N., Son, M.E., Lim, W.C., Lim, S.T., and Cho, H.Y. 2013. Germinated brown rice extract inhibits adipogenesis through the down-regulation of adipogenic genes in 3T3L1 adipocytes. Plant Food Human Nutrition journal. 68(3): 274-278. https://doi.org/ 10.1007/s11130-013-0366-9

Hudak, C.S, and Sul, H.S. 2013. Pref-1, a gatekeeper of adipogenesis. Journal of ndocrinology. 4(79): 1-4. https://doi.org/10.3389/fendo.2013.00079

Huihui, T., Zhang, R., Zhang, M., Li, Q., Wai, Z., Zhang, Y., Tang, X., Deng, Y., and Ma, Y. 2014. Dynamic changes in the free and bound phenolic compounds and antioxidant activity of brown rice at different germination stages. Food Chemistry. 161: 337-344. https://doi.org/10.1016/j.foodchem.2014.04.024

Imam, M.U., and Ismail, M. 2015. An overview on germinated brown rice and its nutrigenomic implications. In Genomics, Proteomics and Metabolomics in Nutraceuticals and Functional Foods. 504-717.

Imam, M.U., Ismail, M., Ooi, D.J., Azmi, N.H., Sarega, N., Chan, K.W., and Bhanger, M.I. 2016. Are bioactive-rich fractions functionally richer? Critical Reviews in Biotechnology. 36(4): 585-93. https://doi.org/10.3109/07388551.2014.995586

Imam, M.U., Musa, S.N.A, Azmi, N.H., and Ismail, M. 2012. Effects of white rice, brown rice and germinated brown rice on antioxidant status of type 2 diabetic rats. International Journal of Molecular Sciences. 13(1): 12952-12969. https://doi.org/10.3390/ ijms131012952

Iwaki, K., and Kitada, Y. 2007. Availability of partially milled rice as a source of $\gamma$-Aminobutyric acid. Food Science. 13(1): 41-47.

Jannoey, P., Niamsup, H., Lumyong, S., Tajima, S., Nomura, M., and Chairote, G. 2010a. $\gamma$-Aminobutyric acid (GABA) accumulations in rice during germination. Chiang Mai Journal Science. 37(1): 124-133.

Jannoey, P., Niamsup, H., Lumyong, S., Suzuki, T., Katayama, T., and Chairote, G. 2010b. Comparison of gamma-aminobutyric acid production in Thai rice grains. World Journal of Microbiology Biotechnology. 26(2): 257-63. https://doi.org/10.1007/s11274-0090168-2

Jing, K., Heo, J.Y., Song, K.S., Seo, K.S., Park, J.H., Kim, J.S., Jung, Y.J., Jo, D.Y., Kweon, G.R., Yoon, W.H., et al. 2009. Expression regulation and function of Pref-1 during adipogenesis of human mesenchymal stem cells (MSCs).Biochimica et Biophysica Acta. 1791(8): 816-826. https://doi.org/10.1016/j.bbalip.2009.04.010 
Karladee, D., and Suriyong, S. 2012. $\gamma$-Aminobutyric acid (GABA) content in different varieties of brown rice during germination. Science Asia. 38: 13-17. https://doi. org/10.2306/scienceasia1513-1874.2012.38.013

Kaukovirta-Norja, A., Willhelmsson, A., and Poutanen, K. 2004. Germination: A means to improve the functionalily of oat. Agricultural Food Science. 13: 100-112.

Lee, J.H., Kim, K.A., Kwon, K.B., Kim, E.K., Lee, Y.R., Song, M.Y., Koo, J.H., Ka, S.O., Park, J.W., and Park, B.H. 2007. Diallyl disulfide accelerates adipogenesis in 3T3L1 cells. International Journal of Molecular Medicine. 20(1): 59-64. https://doi.org/ 10.3892/ijmm.20.1.59

Lim, S.M., Goh, Y.M., Kuan, W.B., and Loh, S.P. 2014. Effect of germinated brown rice extracts on pancreatic lipase, adipogenesis and lipolysis in 3T3-L1 adipocytes. Lipids Health Disease. 13(169): 1-9. https://doi.org/10.1186/1476-511X-13-169

Minatel, I.O., Francisqueti, F.V., Corrêa, C.R., and Lima, G.P.P. 2016. Antioxidant activity of $\gamma$-oryzanol: a complex network of interactions. International Journal of Molecular Science. 17: 1107-1122. https://doi.org/10.3390/ijms17081107

Mohd Esa, N., Abdul Kadir, K.K., Amom, Z., and Azlan, A. 2013. Antioxidant activity of white rice, brown rice and germinated brown rice (in vivo and in vitro) and the effects on lipid peroxidation and liver enzymes in hyperlipidaemic rabbits. Food Chemistry. 141: 1306-1312. https://doi.org/10.1016/j.foodchem.2013.03.086

Moongngarm, A., and Saetung, N. 2010. Comparison of chemical compositions and bioactive compounds of germinated rough rice and brown rice. Food Chemistry. 122(3): 782788. https://doi.org/10.1016/j.foodchem.2010.03.053

Moseti, D., Regassa, A., and Kim, W.K. 2016. Molecular regulation of adipogenesis and potential anti-adipogenic bioactive molecules. International Journal of Molecular Science. 17(124): 1-24. https://doi.org/10.3390/ijms17010124

Nagasaka, R., Chotimarkorn, C., Shafiqul, I.M., Hori, M., Ozaki, H., and Ushio, H. 2007. Anti-inflammatory effects of hydroxycinnamic acid derivatives. Biochemical and Biophysical Research Communications. 358(2): 615-619. https://doi.org/10.1016/j. bbrc.2007.04.178

Naoto, K., Yasu, T., Hiroshi, M., Hiroyuki, T., Toshimasa, Y., Kajuro, K., Shinobu, S., Ryosuke, N., Chikara, I., Takuya, S., et al. 1999. PPAR $\gamma$ mediates high-fat diet-induced adipocyte hypertrophy and insulin resistance. Molecular Cell. 4(4): 597-609. https:// doi.org/10.1016/S1097-2765(00)80210-5

Nicolaysen, A., Gammelsaeter, R., Storm-Mathisen, J., Gundersen, V., and Iversen, P.O. 2007. The components required for amino acid neurotransmitter signaling are present in adipose tissues. Journal of Lipid Research. 48: 2123-2132. https://doi.org/10.1194/ jlr.M700021-JLR200

Ohara, K., Uchida, A., Nagasaka, R., Ushio, H., and Ohshima, T. 2009. The effects of hydroxycinnamic acid derivatives on adiponectin secretion. Phytomedicine. 16: 130137. https://doi.org/10.1016/j.phymed.2008.09.012

Ohara, K., Kiyotani, Y., Uchida, A., Nagasaka, R., Maehara, H., Kanemoto S., Hori, M., and Ushio, H. 2011. Oral administration of $\gamma$-aminobutyric acid and $\gamma$-oryzanol prevents stress-induced hypoadiponectinemia. Phytomedicine. 18: 655-660. https://doi.org/ 10.1016/j. phymed.2011.01.003 
Patil, S.B, and Khan, K. 2011. Germinated brown rice as a value added rice product: A review. Journal of Food Science and Technology. 48(6): 661-667. https://doi.org/10.1007/ s13197-011-0232-4

Paul, T., and Wood, I.S. 2004. Adipokines: inflammation and the pleiotropic role of white adipose tissue. Journal of Nutrition. 92(3): 347-355.

Rupérez, A.I., Gil, A., and Aguilera, C.M. 2014. Genetics of oxidative stress in obesity. International Journal of Molecular Sciences. 15(1): 3118-3144. https://doi.org/10.3390/ ijms 15023118

Sfar, S., Boussoffara, R., Sfer, M.T., and Kerkeni, A. 2013. Antioxidant enzymes activities ib obese Tunisian children. Nutrition Journal. 12(1): 18-25. https://doi.org/10.1186/14752891-12-18.

Teng, K.T., Chang, C.Y., Chang, L.F., and Nesaretnam, K. 2014. Modulation of obesityinduced inflammation by dietary fats: mechanisms and clinical evidence. Journal of Nutrition. 13: 12. https://doi.org/10.1186/1475-2891-13-12

Tilg, H., and Moschen, A.R. 2008. Inflammatory mechanisms in the regulation of insulin resistance. Molecular Medicine. 14(3-4): 222-231. https://doi.org/10.2119/200700119.Tilg

Wang, Y., Hudak, C., and Sul, H.S. 2010. Role of preadipocyte factor 1 in adipocyte differentiation. Clinical Lipidology. 5(1): 109-115. https://doi.org/10.2217/clp.09.80

Wihong, P., Songsri, P. Suriharn, B. Lomthaisong, K., and Lertrat, K. 2014. Lycopene and beta-carotene contents in different spiny bitter gourd (Momordica cochinchinensis (Lour.) Spreng) clones. Khon Kaen Agriculture Journal. 42(1): 1.

Wu, Z., Bucher, N.L., and Farmer, S.R. 1996. Induction of peroxisome proliferator-activated receptor $\mathrm{g}$ during the conversion of $3 \mathrm{~T} 3$ fibroblasts into adipocytes is mediated by $\mathrm{C} / \mathrm{EBP} \beta, \mathrm{C} / \mathrm{EBP} \alpha$, and glucocorticoids. Molecular and cellular biology. 16(8): 41284136.

Zhao, H., Han, Z., Ji, X., and Luo, Y. 2016. Epigenetic regulation of oxidative stress in ischemic stroke. Aging Disease. 7(3): 295-306. https://doi.org/10.14336/AD.2015.1009 